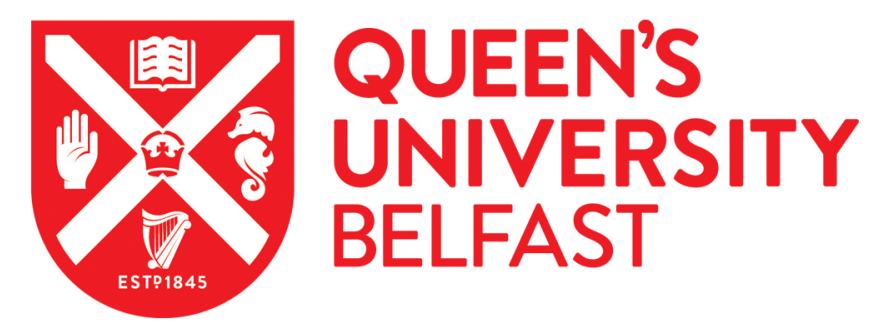

\title{
Coherent and stochastic contributions of compound resonances in atomic processes: Electron recombination, photoionization, and scattering
}

Flambaum, V. V., Kozlov, M. G., \& Gribakin, G. F. (2015). Coherent and stochastic contributions of compound resonances in atomic processes: Electron recombination, photoionization, and scattering. Physical Review $A$ (Atomic, Molecular, and Optical Physics), 91(5), [052704]. https://doi.org/10.1103/PhysRevA.91.052704

Published in:

Physical Review A (Atomic, Molecular, and Optical Physics)

Document Version:

Peer reviewed version

Queen's University Belfast - Research Portal:

Link to publication record in Queen's University Belfast Research Portal

Publisher rights

(c) 2015 American Physical Society.

\section{General rights}

Copyright for the publications made accessible via the Queen's University Belfast Research Portal is retained by the author(s) and / or other copyright owners and it is a condition of accessing these publications that users recognise and abide by the legal requirements associated with these rights.

Take down policy

The Research Portal is Queen's institutional repository that provides access to Queen's research output. Every effort has been made to ensure that content in the Research Portal does not infringe any person's rights, or applicable UK laws. If you discover content in the Research Portal that you believe breaches copyright or violates any law, please contact openaccess@qub.ac.uk. 


\title{
Coherent and stochastic contributions of compound resonances in atomic processes: Electron recombination, photoionization and scattering
}

\author{
V. V. Flambaum,,$^{1,2}$ M. G. Kozlov, ${ }^{1,3,4}$ and G. F. Gribakin ${ }^{5}$ \\ ${ }^{1}$ School of Physics, University of New South Wales, Sydney 2052, Australia \\ ${ }^{2}$ New Zealand Institute for Advanced Study, Massey University Auckland, 0745 Auckland, New Zealand \\ ${ }^{3}$ Petersburg Nuclear Physics Institute, Gatchina 188300, Russia \\ ${ }^{4}$ St Petersburg Electrotechnical University "LETI", Prof Popov St 5, St Petersburg 197376, Russia \\ ${ }^{5}$ School of Mathematics and Physics, Queen's University Belfast, \\ Belfast BTr 1 NN, Northern Ireland, United Kingdom
}

(Dated: May 13, 2015)

\begin{abstract}
In open-shell atoms and ions, processes such as photoionization, combination (Raman) scattering, electron scattering and recombination, are often mediated by many-electron compound resonances. We show that their interference (neglected in the independent-resonance approximation) leads to a coherent contribution, which determines the energy-averaged total cross sections of electron- and photon-induced reactions obtained using the optical theorem. In contrast, the partial cross sections (e.g., electron recombination, or photon Raman scattering) are dominated by the stochastic contributions. Thus, the optical theorem provides a link between the stochastic and coherent contributions of the compound resonances. Similar conclusions are valid for reactions via compound states in molecules and nuclei.
\end{abstract}

PACS numbers: $31.10 .+\mathrm{z}, 34.10 .+\mathrm{x}, 34.80 . \mathrm{Lx}, 05.45 . \mathrm{Mt}$

\section{INTRODUCTION}

The aim of this paper is to examine the interplay between simple, singly or doubly excited "doorway" states, and multiply excited chaotic eigenstates (compound resonances) in processes, such as photon and electron scattering, photoionization, electron recombination, etc., involving complex atomic or molecular systems. In particular, we identify the coherent and incoherent contributions of the compound resonances, and show how these are related to the total and partial cross sections of various reactions. We outline a method for the calculation of probabilities of these reactions, which involves summations over the doorway states, rather than the eigenstates.

\section{A. Many-body quantum chaos}

Consider a finite quantum system with many degrees of freedom, such as a many-electron atom or ion, a polyatomic molecule, or a heavy nucleus. In the zerothorder approximation, the states of such system can be constructed from some single-particle states. For atoms these will be the electron orbitals obtained in some meanfield potential, e.g., using the Hartree-Fock method. For molecular vibrations, the zeroth-order states are normalmode vibrations, which are determined by the quadratic expansion of the ground-state electronic energy near the equilibrium positions of the nuclei.

In general, this description works well for the ground state of the system, and in many cases, it also provides a correct picture of low-lying excitations. Thus, the ground states of most atoms and ions are characterized by their electronic configuration. The ground state of the molec- ular vibrational Hamiltonian is simply a product of the zero-point motion states of all the normal modes. Lowenergy excitations then correspond to promotions of one of the electrons into an excited-state orbital, or adding a vibrational quantum to one of the normal-mode harmonic oscillators.

Of course, the exact energy of the atomic excitation will be affected by the residual two-body Coulomb interaction between the electrons. Such correction can be relatively small in atoms or ions with a simple ground-state configuration (e.g., in alkali-like systems with one active electron above a closed-shell core). At the same time, in systems with several valence electrons, and in particular, with open-shell ground-state configurations, the singleparticle picture does not hold well at all. A state in which one of the electrons is promoted to a higher-lying orbital will be mixed with other excited states, in which two or three electrons have changed their places. Such effects are usually described as configuration mixing. Finding the eigenstates of the system then requires constructing a basis of many-electron states of the relevant electronic configurations, and diagonalizing the Hamiltonian of the residual interaction in this basis. Similarly, accurate vibrational energies can be found by including anharmonic, e.g., cubic and quartic, terms in the vibrational Hamiltonian and diagonalizing its matrix, constructed using the zeroth-order (harmonic) basis states.

A practical limitation to this approach is set by the maximum size of a matrix that can be diagonalized efficiently on a computer. The Hamiltonian matrix sizes become very large in atomic systems with open $d$ and $f$ shells, due to a large number of active electrons, or in polyatomic molecules with many vibrational degrees of freedom. Such systems are characterized by large densities of the energy spectra, which promotes strong mixing 
of the zeroth-order basis states. As a result, each of the eigenstates becomes a superposition of a large number of basis states, with the expansion coefficients behaving like random variables. Further, these eigenstates often cannot be assigned any meaningful quantum numbers, except the exact ones, such as the total angular momentum or parity. Even when the Hamiltonian matrix sizes are manageable, "exact" calculations of the spectra and processes in such systems are virtually impossible due to the extreme sensitivity of the eigenvalues to small perturbations, e.g., the effect of states omitted from the basis, or higher-order corrections to the perturbation.

This behaviour of quantum systems is termed quantum chaos. Besides the Gaussian statistics of the eigenstate components, there is also a specific correlation between the energy eigenvalues, which is characteristic of the spectra of random matrices [1]. Well-known examples of quantum-chaotic systems are excited heavy nuclei (e.g., those formed by neutron capture) $[2,3]$, and heavy atoms and ions with open $f$ shells, such as Ce or $\mathrm{Au}^{24+}[4-6]$. Another example is given by the vibrational motion of polyatomic molecules where anharmonic mixing between normal modes leads to intramolecular vibrational redistribution (IVR) [7-10]. Chaotic resonances have also been found recently in ultracold collisions of erbium atoms [11] (a manifestation of chaotic states in the excited $\mathrm{Er}_{2}$ molecule).

In each of these examples the quantum-chaotic behaviour of the system leads to important observable effects beyond the energy-level statistics. Narrowly spaced neutron resonances in heavy nuclei provide strong enhancements of parity nonconservation due to the weak interaction $[12,13]$. Electron capture in chaotic multielectronic resonances in open-shell ions results in recombination rates $10^{2}-10^{3}$ times greater than the singleparticle radiative recombination rate $[5,14-16]$, as seen in many experiments [17-22]. Similar states feature in photoionization and photoemission in many ions, producing a complex interplay of broad and narrow resonances [23-29]. IVR is an essential step in most chemical reactions. It also plays a key role in electron attachment and positron annihilation in polyatomic molecules [30, 31].

\section{B. Doorway states}

While the exact calculation of many-body chaotic eigenstates is impossible, their nature allows one to develop a statistical theory to calculate the mean-squared values of matrix elements and amplitudes involving such states $[4,13,32-37]$. In this way one can predict observables averaged over a small energy interval containing many such states (which is often sufficient since the individual states cannot be resolved experimentally).

Of particular importance in this approach are doorway states. A doorway is a state which is coupled in the lowest order to the initial state. For example, in electronion recombination, doorways are dielectronic states (i.e., 1-hole-2-particle excitations of the combined ion). In molecular infrared photoabsorption, doorways are singlemode excitations. In considering the IVR process of a single-mode excitations, the doorways are two- and threequantum vibrational excitations coupled to the singlemode excitation in the lowest order.

To illustrate these examples, Fig. 1 (a) shows schematically the absorption of an infrared photon of energy $\omega$ by a polyatomic molecule, followed by IVR. Figure 1 (b) describes the recombination of an electron with energy $\varepsilon$ with a multicharged positive ion $A^{q+}$.

In the description of both processes, we adopt a temporal picture of the dynamics, as if probed by a short initial pulse. This picture is observed directly in the pump-probe studies of molecular IVR [38]. In contrast, the electron-ion recombination usually deals with incident electrons of definite energy. (In spite of the high energy resolution achieved in experiments with electron coolers in ion storage rings $[18,20,39,40]$, the measurements for complex targets are incapable of resolving individual chaotic resonances [41].)

\section{Vibrational excitation of molecules}

In the process shown in Fig. 1 (a), the energy of the photon is tuned to the frequency of the normal mode 1 (e.g., a $\mathrm{CH}$ or $\mathrm{OH}$ stretch mode, with $\omega \sim 3000 \mathrm{~cm}^{-1}$ ). In the first step the photon excites a single-quantum vibration of this mode. Lowest-order (cubic and quartic) anharmonic couplings $V_{\text {anh }}$ perturbatively couple this initial state to some two- or three-mode vibrational excitations. These off-resonance states act as "doorways" which mediate the spreading of the vibrational energy into more complex multi-mode vibrational states, whose density is much higher than that of the modes or doorways. If $V_{\text {anh }}$ is sufficiently strong (and suitable doorway states are available), the excitation ultimately spreads into the "bath" of closely-spaced states [42].

This is the essence of the IVR process. Its time scale $\tau \sim \hbar / \Gamma_{\text {IVR }}$, is related to the energy width of the initial single-mode state with respect to its decay towards the bath states. High-resolution molecular spectroscopy in fact allows one to observe these states as clumps of narrowly spaced absorption lines within $\Gamma_{\text {IVR }}$ energy interval of the vibrational fundamental [43]. The number of such lines is $N \sim \Gamma_{\text {IVR }} \rho_{v}$, where $\rho_{v}$ is the total density of the vibrational spectrum for a given symmetry, at this energy.

\section{Electron-ion recombination}

Turning to the second process [Fig. 1 (b)], the ground state of the target ion $A^{q+}$ is usually a simple state described by a single dominant electronic configuration. The Coulomb interaction $V$ between the incident and target electrons couples the initial state $\left(e^{-}+A^{q+}\right)$ to 


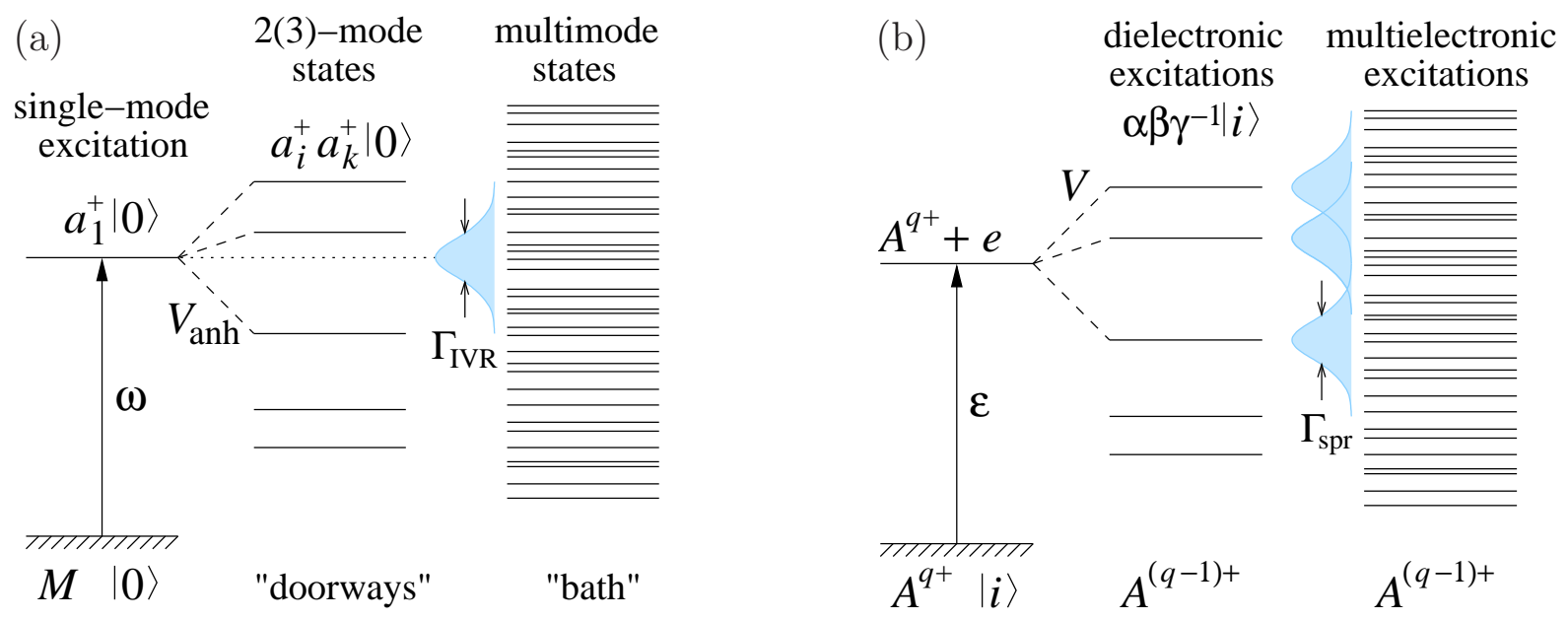

FIG. 1. Interplay between simple, single-particle degrees of freedom and multiply excited states in the infrared photoabsorption by a polyatomic molecule (a) and electron recombination with a many-electron open-shell ion (b) (see text for details).

the doubly excited states of the compound ion $A^{(q-1)+}$. In such states two electrons occupy some excited-state orbitals $(\alpha$ and $\beta)$, leaving a hole in one of the target ground-state orbitals $(\gamma)$. For simple targets, photoemission from the doubly excited state completes the dielectronic recombination process [44].

For open-shell targets such as $\mathrm{Au}^{25+}$ (with $4 f^{8}$ outer orbital ground-state configuration), the dielectronic resonances are embedded in a dense spectrum of multiply excited states and are strongly mixed with them $[5,6]$. In the temporal picture this mixing describes a rapid decay of the dielectronic excited states into chaotic "compound states" (a term which originated in nuclear physics, sometimes called Feshbach resonances [45]). Its time constant $\tau$ is determined by the so-called spreading width $\Gamma_{\mathrm{spr}}$, as $\tau=\hbar / \Gamma_{\mathrm{spr}}$. (It plays the same role as $\Gamma_{\text {IVR }}$ in the first example, but on a completely different scale, e.g., $\Gamma_{\mathrm{spr}} \sim 10 \mathrm{eV}$ in $\mathrm{Au}^{24+}$.) In the energy eigenstate picture, each of the dielectronic states appears as a component in many chaotic compound states, contributing significantly to $N \sim \Gamma_{\mathrm{spr}} / D$ of them ( $D$ being the small level spacing between the compound states).

As a result of this spreading, the weight of every doorway in a given compound state is $\sim 1 / N$, and the probability for the compound states to autoionize (i.e., re-emit the electron) is greatly reduced $\left(\propto N^{-1}\right)$. On the other hand, their lifetimes with respect to emitting a photon are similar to those of the dielectronic (and singly excited) states, since any excited electron in the compound state can radiate. The electron "trapping" in the chaotic compound states thus leads to strongly increased recombination rates $[5,14]$. Specific examples of doorway states for electron recombination with $\mathrm{Au}^{25+}$ can be found in Table I of Ref. [14], while mixing of doorways with chaotic states was explored in Ref. [6].

The energy spacing between the compound states can be very small (see, e.g., the estimates for $\mathrm{Au}^{24+}$ in Refs. $[5,14])$, beyond the best resolution available in the recom- bination experiments [41]. This does not mean, however, that the recombination cross section is completely structureless. The dielectronic doorway states can produce broad maxima with widths $\sim \Gamma_{\text {spr }}$ in the energy dependence of the cross section. This is similar to the way in which the frequencies and strengths of vibrational fundamentals determine the overall infrared absorption spectrum of a polyatomic molecule. Here the normal modes excited by the photon play the role of doorways for the IVR which follows molecular photoabsorption.

In what follows we consider a variety of processes initiated by a photon or electron impact on a complex atomic or molecular system. We aim to determine the roles played by the simple doorway states and chaotic, compound states in each case. Although most of the expressions and conclusions are quite general, we will use the language of atoms (or ions) and atomic processes, with the many degrees of freedom and complexity (chaos) arising from the large numbers of active electrons and available orbitals.

\section{THEORY}

\section{A. Compound states.}

In isolated quantum many-body systems chaos emerges due to a rapid, exponential growth of the level density with energy. This growth is caused by the increase in the number of active particles promoted into unoccupied orbitals, following the increase in the excitation energy of the system. When the residual interaction between the particles is greater than the energy spacing between the levels that it mixes, the eigenstates $|n\rangle$ become chaotic superpositions of the basis states $|b\rangle$, constructed from the single-particle orbitals (e.g., Slater determinants, for the Fermi system). In this regime the coefficients in the 
eigenstate expansion,

$$
|n\rangle=\sum_{b} C_{b}^{(n)}|b\rangle
$$

behave as uncorrelated random variables:

$$
\overline{C_{b}^{(n)}}=0, \quad \overline{C_{a}^{(m)} C_{b}^{(n)}}=\delta_{m n} \delta_{a b} \overline{C_{b}^{(n)^{2}}} .
$$

Note that we use indices $m, n$, etc., to denote the compound eigenstates, and $a, b$, etc., for the basis states, and the averages are taken over nearby eigenstates.

For the system under consideration the Hamiltonian matrix $H_{a b}$ and the coefficients $C_{b}^{(n)}$ can be made real. We also assume that the basis states and the eigenstates have the same exact quantum numbers (e.g., the total angular momentum and parity, for a spherically symmetric system), and the usual normalization condition applies: $\sum_{b}\left|C_{b}^{(n)}\right|^{2}=\sum_{n}\left|C_{b}^{(n)}\right|^{2}=1$.

Besides Eq. (2), the coefficients display a systematic dependence on the eigenstate energy. This dependence can be described by (see, e.g., [4]),

$$
\overline{C_{b}^{(n)^{2}}}=\frac{D}{2 \pi} \frac{\Gamma_{\mathrm{spr}}}{\left(E_{n}-E_{b}\right)^{2}+\Gamma_{\mathrm{spr}}^{2} / 4},
$$

where $E_{n}$ is the energy eigenvalue of state $n, E_{b} \equiv H_{b b}$ is the expectation energy of the basis state $b$, and $D$ is the mean energy spacing between the eigenstates. The parameter $\Gamma_{\mathrm{spr}}$ is the spreading width. It characterizes the size of the energy interval in which the typical coefficients are close to maximum, $C_{b}^{(n)} \sim 1 / \sqrt{N}$, where $N=\pi \Gamma_{\mathrm{spr}} /(2 D)$, is the number of principal components, i.e., the number of basis states that contribute significantly to a given eigenstate. In the strong mixing regime, $\Gamma_{\text {spr }} \gg D$ and $N \gg 1$. The spreading width can be calculated using the Golden Rule as $\Gamma_{\mathrm{spr}}=2 \pi \overline{\left|H_{a b}\right|^{2}} / D_{b}$, where $D_{b}$ is the mean spacing between the states $b$ to which a given basis state $a$ is coupled [15], or evaluated in relatively small scale configuration-interaction calculations [6]. Its values in atomic systems range from $\sim 1 \mathrm{eV}$ in atoms, such as Ce [4], to $\sim 10 \mathrm{eV}$ in multicharged ions, e.g., $\mathrm{Au}^{24+}[5,6]$, or $\mathrm{W}^{q+}(q=18-24)[15]$.

\section{B. Coherent amplitudes}

Let us consider the process of photoexcitation of a many-electron atom or ion $A$ from the ground state $|0\rangle$ to an excited state $|n\rangle$ above the ionization limit. This leads to either autoionization $\left(A+\gamma \rightarrow A^{+}+e\right)$ or radiative quenching of the excited state $\left(A+\gamma \rightarrow A^{*}+\gamma^{\prime}\right)$. The corresponding amplitudes are

$$
\begin{aligned}
M_{k \varepsilon}^{\gamma e} & =\sum_{n} \frac{\langle k, \varepsilon|\hat{V}| n\rangle\langle n|\hat{D}| 0\rangle}{E_{0}+\omega-E_{n}+\frac{i}{2} \Gamma_{n}}, \\
M_{m}^{\gamma \gamma^{\prime}} & =\sum_{n} \frac{\langle m|\hat{D}| n\rangle\langle n|\hat{D}| 0\rangle}{E_{0}+\omega-E_{n}+\frac{i}{2} \Gamma_{n}},
\end{aligned}
$$

where $\hat{D}$ is the electron-photon interaction operator, $\omega$ is the photon energy, and $\hat{V}$ is the electron Coulomb interaction. The first amplitude corresponds to the final state $|k\rangle$ of the ion $A^{+}$and an electron in the continuum state $|\varepsilon\rangle$. The second amplitude describes photon (Raman) scattering leading to the final atomic state $m$ and a photon $\gamma^{\prime}$. The sums are over the compound eigenstates $n$ with the energy $E_{n}$ and total width $\Gamma_{n}$ (due to both autoionization and radiative decay).

Note that in considering the photon impact we neglect the possibility of direct electron emission into the continuum. Such process will either produce a distinct, smooth background for the resonant contributions, or, more likely for complex targets, the continuum states will be strongly mixed with the autoionizing resonances [46].

Using Eq. (1) in Eq. (4), and averaging this amplitude over a small energy interval containing many compound states $n$, gives the coherent part of the photoionization amplitude:

$$
\overline{M_{k \varepsilon}^{\gamma e}}=\sum_{n d} \overline{C_{d}^{(n)^{2}}} \frac{\langle k \varepsilon|\hat{V}| d\rangle\langle d|\hat{D}| 0\rangle}{E_{0}+\omega-E_{n}+\frac{i}{2} \Gamma_{n}},
$$

where we also made use of Eq. (2). The sum in Eq. (6) is over the compound states $n$ and basis states $d$. Since $\hat{D}$ is a one-body operator, the matrix element $\langle d|\hat{D}| 0\rangle$ is nonzero only for the basis states $d$ in which one of the ground-state electrons is excited by the photon (assuming that the ground state has a well-defined configuration). Such states $d$ play the role of doorway states for the resonant photoabsorption process.

The mean spacing $D$ between the compound resonances is very small, which allows one to replace summation over $n$ by integration,

$$
\sum_{n} \longrightarrow \int \frac{d E_{n}}{D}
$$

Using Eq. (3) in Eq. (6), we then obtain

$$
\overline{M_{k \varepsilon}^{\gamma e}}=\sum_{d} \frac{\langle k, \varepsilon|\hat{V}| d\rangle\langle d|\hat{D}| 0\rangle}{E_{0}+\omega-E_{d}+\frac{i}{2} \Gamma_{\mathrm{spr}}},
$$

where $\Gamma_{n} \ll \Gamma_{\text {spr }}$ has been assumed. The latter relation is supported by numerical calculations [5, 6, 14-16], which show that the natural width of compound states $\Gamma_{n}$ is several orders of magnitude smaller than $\Gamma_{\text {spr }}$. Similarly, averaging the amplitude in Eq. (5) gives

$$
\overline{M_{m}^{\gamma \gamma^{\prime}}}=\sum_{d} \frac{\langle m|\hat{D}| d\rangle\langle d|\hat{D}| 0\rangle}{E_{0}+\omega-E_{d}+\frac{i}{2} \Gamma_{\mathrm{spr}}} .
$$

Equations (8) and (9) reveal the physical meaning of the coherent amplitudes. They describe the excitation of the system into simple doorway states $d$, which then decay directly into the final states. (In the incoherent, "stochastic" contribution, the capture into a compound state $n$ and its decay are due to different basis-state components $d$ and $e$, see Sec. IID.) Doorway states are not 
the eigenstates of the Hamiltonian, as they are mixed by the Coulomb interaction with other basis states with two, three and more excited electrons. In the temporal picture of the process, the photon initially excites one electron. This is followed by a chain of electron interactions, until all the excitation energy is shared between as many electrons as possible (cf. Fig. 1). This internal decay of the doorway state on the time scale $\sim \hbar / \Gamma_{\text {spr }}$ explains the origin of the spreading width in the denominators of Eqs. (8) and (9). The spreading width $\Gamma_{\text {spr }}$ is similar to the quasiparticle width in a solid where quasiparticles also decay into internal excitations of the solid (see, e.g., Ref. [47] and references therein).

The doorway states for photoionization are singleelectron excitations from the ground state. The eigenstates of the Hamiltonian (i.e., the compound resonances) contribute coherently to each doorway state. Therefore, this contribution is not included in the standard independent-resonance approximation [48, 49].

\section{Total cross section}

The total cross section of the photon- or electroninduced reactions, averaged over the compound resonances, can be found using the optical theorem [48], from the elastic forward-scattering amplitude, e.g., for the photon-induced case, $\sigma_{\text {tot }}^{\gamma} \propto \operatorname{Im} M_{0}^{\gamma \gamma}$. Averaging this relation over the compound resonances involves the coherent contribution (9) for $|m\rangle=|0\rangle$, and we have

$$
\sigma_{\text {tot }}^{\gamma} \propto \operatorname{Im} \overline{M_{0}^{\gamma \gamma}}=\frac{1}{2} \sum_{d} \frac{|\langle d|\hat{D}| 0\rangle|^{2} \Gamma_{\mathrm{spr}}}{\left(E_{0}+\omega-E_{d}\right)^{2}+\Gamma_{\mathrm{spr}}^{2} / 4},
$$

where the sum is over the doorway states $d$.

Note that the integral contribution of each of the doorway states in Eq. (10) $\left(\int \sigma_{\text {tot }} d \omega\right)$ is independent of $\Gamma_{\mathrm{spr}}$. The total photoabsorption cross section is given by the sum of the single-particle (i.e., doorway) contributions. The only manifestation of the strong mixing and chaotic dynamics in the system is the broadening of these singleparticle peaks by $\Gamma_{\mathrm{spr}}$ (which is much greater than the natural widths of the single-particle excitations).

A familiar example of this picture is the infrared absorption spectra of molecules, which are dominated by characteristic peaks of various modes. A low-resolution measurement of the total cross section will not reveal any features related to the strong mixing or IVR, which take place after the absorption of the photon.

As a consistency check we can obtain the result of Eq. (10) starting from the sum over compound states in Eq. (5). Setting $m=0$, we have

$$
\operatorname{Im} M_{0}^{\gamma \gamma}=\frac{1}{2} \sum_{n} \frac{|\langle n|\hat{D}| 0\rangle|^{2} \Gamma_{n}}{\left(E_{0}+\omega-E_{n}\right)^{2}+\Gamma_{n}^{2} / 4} .
$$

Using Eqs. (1) and (2), one obtains

$$
\operatorname{Im} \overline{M_{0}^{\gamma \gamma}}=\frac{1}{2} \sum_{n d} \overline{C_{d}^{(n)^{2}}} \frac{|\langle d|\hat{D}| 0\rangle|^{2} \Gamma_{n}}{\left(E_{0}+\omega-E_{n}\right)^{2}+\Gamma_{n}^{2} / 4},
$$

and applying Eqs. (3) and (7) again leads to Eq. (10). One can also obtain Eq. (10) by averaging Eq. (11) over a photon energy interval $\Delta \omega, \Gamma_{n} \ll \Delta \omega \ll \Gamma_{\text {spr }}$, containing a large number of resonances $\Delta \omega / D$ (i.e., integrating the resonant contributions over $\omega$ instead of $E_{n}$ ).

A calculation similar to that in Sec. II B, yields coherent amplitudes of the electron-induced processes, i.e., photorecombination $\left(A^{q+}+e \rightarrow A^{(q-1)+}+\gamma\right)$ and electron scattering $\left(A^{q+}+e \rightarrow A^{q+^{*}}+e^{\prime}\right)$ via compound resonances:

$$
\begin{aligned}
& \overline{M_{i m}^{e \gamma}}=\sum_{d} \frac{\langle m|\hat{D}| d\rangle\langle d|\hat{V}| i, \varepsilon\rangle}{E_{i}+\varepsilon-E_{d}+\frac{i}{2} \Gamma_{\mathrm{spr}}}, \\
& \overline{M_{i k}^{e e^{\prime}}}=\sum_{d} \frac{\left\langle k, \varepsilon^{\prime}|\hat{V}| d\right\rangle\langle d|\hat{V}| i, \varepsilon\rangle}{E_{i}+\varepsilon-E_{d}+\frac{i}{2} \Gamma_{\mathrm{spr}}} .
\end{aligned}
$$

Here the doorway states $d$ are dielectronic excitations of the ion $A^{(q-1)+}$, produced by capturing the incident electron simultaneously with excitation of an electron of the target $A^{q+}$. In Eqs. (13) and (14), $i$ is the initial (e.g., ground) state of the target ion, $m$ is the final state of the ion $A^{(q-1)+}$, and $k$ is the final state of $A^{q+^{*}}$.

In analogy to Eq. (10), the averaged total resonant electron-impact cross section is

$$
\sigma_{\text {tot }}^{e} \propto \operatorname{Im} \overline{M_{i i}^{e e}}=\frac{1}{2} \sum_{d} \frac{|\langle d|\hat{V}| i, \varepsilon\rangle|^{2} \Gamma_{\mathrm{spr}}}{\left(E_{i}+\varepsilon-E_{d}\right)^{2}+\Gamma_{\mathrm{spr}}^{2} / 4} .
$$

It describes all processes following the capture of an electron in the dielectronic doorway states, broadened (via $\left.\Gamma_{\mathrm{spr}}\right)$ by multiconfigurational mixing which defines the compound eigenstates.

Equations (10) and (15) can be written in the familiar Breit-Wigner form by replacing the squared matrix elements by the corresponding partial widths for the decay of the doorway state. Hence, we introduce the radiative width $\Gamma_{d \rightarrow 0}^{(r)} \propto|\langle d|\hat{D}| 0\rangle|^{2}$, and the autoionization width $\Gamma_{d \rightarrow i}^{(a)} \propto|\langle d|\hat{V}| i, \varepsilon\rangle|^{2}$. It is also natural to add the total radiative width $\Gamma_{d}^{(r)}$ and total autoionization width $\Gamma_{d}^{(a)}$ of the doorway to its spreading width, to account for all decay modes of this state. The total width of the doorway state then is $\Gamma_{d}=\Gamma_{\mathrm{spr}}+\Gamma_{d}^{(r)}+\Gamma_{d}^{(a)}$, and the cross sections are given by

$$
\begin{aligned}
& \sigma_{\text {tot }}^{\gamma} \propto \sum_{d} \frac{\Gamma_{d \rightarrow 0}^{(r)} \Gamma_{d}}{\left(E_{0}+\omega-E_{d}\right)^{2}+\Gamma_{d}^{2} / 4}, \\
& \sigma_{\text {tot }}^{e} \propto \sum_{d} \frac{\Gamma_{d \rightarrow i}^{(a)} \Gamma_{d}}{\left(E_{0}+\omega-E_{d}\right)^{2}+\Gamma_{d}^{2} / 4} .
\end{aligned}
$$

In this form it is easy to restore the correct prefactor in these equations, by comparison with the standard BreitWigner formula [48]. 
In Sec. II B and above, the doorways states were introduced as particular types of basis states selected by the process under consideration. To make Eqs. (10) and (15) [or (16) and (17)] more accurate for application to real systems, one can diagonalize the Hamiltonian matrix in the subspace of the doorway states. This should supply more accurate energies $E_{d}$ and amplitudes involving the doorways. In complex systems the doorways are only a small part of the total Hilbert space in the energy range of interest, making this task feasible.

Note that Eqs. (16) and (17) provide interpolation formulas for the total cross sections. They can describe a transition from the chaotic compound resonance regime, in which $\Gamma_{d} \approx \Gamma_{\mathrm{spr}}$, to the simple resonance regime $\Gamma_{d} \approx \Gamma_{d}^{(r)}+\Gamma_{d}^{(a)}$ (in which the "doorway" states do not spread). For $\Gamma_{d}^{(a)}+\Gamma_{d}^{(r)} \gg \Gamma_{\text {spr }}$, the doorway state has no time to excite other electrons and is decoupled from the compound resonances. This can also be explained using perturbation theory. In this case the energy difference between a doorway state $d$ and a compound state $n$, $E_{d}-E_{n}-i\left(\Gamma_{d}^{(a)}+\Gamma_{d}^{(r)}\right) / 2$, is dominated by the imaginary part and becomes larger than the the matrix element of the residual interaction $\hat{V}$, which can mix $d$ and $n$, i.e., $\langle n|\hat{V}| d\rangle / \Gamma_{d}^{(a)} \ll 1$. (Except for the very highly charged ions, $\Gamma_{d}^{(a)} \gg \Gamma_{d}^{(r)}$ for the dielectronic states.)

Numerical calculations for $\mathrm{W}^{19+}$ and $\mathrm{Au}^{24+}$ show that $\Gamma_{d}^{(a)} \ll \Gamma_{\mathrm{spr}}$, and the electron recombination processes in such ions are dominated by the many-electron compound resonances [14-16] (see below).

\section{Partial cross sections}

The total width of a resonance $n$ is the sum of its partial widths over all final states or decay channels, $\Gamma_{n}=\sum_{f} \Gamma_{n}^{(f)}$. In the independent-resonance approximation the partial cross section $\sigma_{f}$ for channel $f$ (averaged over the resonances) can be obtained by multiplying the total cross section $\sigma_{\text {tot }}$ by the average ratio of the corresponding partial width $\Gamma_{n}^{(f)}$ to the total width $\Gamma_{n}$. In most cases the compound state $n$ can decay into many final states, which suppresses the fluctuations of $\Gamma_{n}\left[14^{-}\right.$ 16], and one obtains

$$
\sigma_{f} \approx \sigma_{\mathrm{tot}} \overline{\Gamma_{n}^{(f)}} / \overline{\Gamma_{n}}
$$

However, in this approximation one misses a specific, coherent contribution to the partial cross section, which is calculated below.

The resonance-averaged cross section (or probability) of a process is proportional to the modulus squared amplitude, $P=\overline{|M|^{2}}$. When analyzing this quantity, it is convenient to separate out the coherent term, $P_{\text {coh }}=$ $|\bar{M}|^{2}$. The remaining part then represents the stochastic contribution: $P_{\text {sto }}=\overline{|M|^{2}}-|\bar{M}|^{2}$.

Let us consider photoionization as an example. The corresponding resonance-averaged probability $P^{\gamma e}$ is found by taking the squared modulus of the amplitude $M_{k \varepsilon}^{\gamma e}$ from Eq. (4):

$$
\left|M_{k \varepsilon}^{\gamma e}\right|^{2}=\sum_{n, n^{\prime}} \frac{\left\langle 0|\hat{D}| n^{\prime}\right\rangle\left\langle n^{\prime}|\hat{V}| k, \varepsilon\right\rangle}{E_{0}+\omega-E_{n^{\prime}}-\frac{i}{2} \Gamma_{n^{\prime}}} \frac{\langle k, \varepsilon|\hat{V}| n\rangle\langle n|\hat{D}| 0\rangle}{E_{0}+\omega-E_{n}+\frac{i}{2} \Gamma_{n}}
$$

Each of the four matrix elements in this expression involves one compound state $\left(n\right.$ or $\left.n^{\prime}\right)$, which can be expanded as in Eq. (1). After this, averaging of Eq. (19) reduces to finding the averaged product of four expansion coefficients:

$$
\overline{C_{a}^{\left(n^{\prime}\right)} C_{b}^{\left(n^{\prime}\right)} C_{c}^{(n)} C_{d}^{(n)}}=\delta_{a b} \overline{C_{b}^{\left(n^{\prime}\right)^{2}}} \delta_{c d} \overline{C_{d}^{(n)^{2}}}+\delta_{n^{\prime} n} \delta_{a d} \overline{C_{d}^{(n)^{2}}} \delta_{b c} \overline{C_{b}^{(n)^{2}}}+\delta_{n^{\prime} n} \delta_{a c} \overline{C_{a}^{(n)^{2}}} \delta_{b d} \overline{C_{d}^{(n)^{2}}}
$$

which follows from Eq. (2). Hence, the average of Eq. (19) is the sum of three distinct terms:

$$
P^{\gamma e}=\left|\sum_{n d} \overline{C_{d}^{(n)^{2}}} \frac{\langle k, \varepsilon|\hat{V}| d\rangle\langle d|\hat{D}| 0\rangle}{E-E_{n}+\frac{i}{2} \Gamma_{n}}\right|^{2}+\sum_{n b d} \overline{C_{b}^{(n)^{2}}} \overline{C_{d}^{(n)^{2}}} \frac{|\langle k, \varepsilon|\hat{V}| b\rangle|^{2}|\langle d|\hat{D}| 0\rangle|^{2}}{\left(E-E_{n}\right)^{2}+\Gamma_{n}^{2} / 4}+\sum_{n}\left|\sum_{d} \overline{C_{d}^{(n)^{2}}} \frac{\langle d|\hat{V}| k, \varepsilon\rangle\langle d|\hat{D}| 0\rangle}{E-E_{n}-\frac{i}{2} \Gamma_{n}}\right|^{2},
$$

where $E=E_{0}+\omega$ is the total energy of the system.

The first term on the right hand side of Eq. (21) is the coherent contribution, cf. Eq. (6). The second term corresponds to the independent resonance approximation, and is usually the only term considered [48, 49]. The weights given by the mean-squared coefficients, which multiply the modulus squared matrix elements for au- toionization and photoabsorption, link the corresponding 
partial widths of the compound and doorway states:

$$
\begin{aligned}
& \Gamma_{n \rightarrow 0}^{(r)}=\sum_{d} \overline{C_{d}^{(n)^{2}}} \Gamma_{d \rightarrow 0}^{(r)}, \\
& \Gamma_{n \rightarrow k}^{(a)}=\sum_{b} \overline{C_{b}^{(n)^{2}}} \Gamma_{b \rightarrow k}^{(a)}
\end{aligned}
$$

The last term in Eq. (21) is the remaining part of the stochastic contribution, and we call it the residual stochastic term. The stochastic contribution thus consists of the independent resonance (IR) contribution and the residual stochastic term, $P_{\text {sto }}=P_{\mathrm{IR}}+P_{\text {res }}$.

Using Eqs. (3) and (7) [or averaging Eq. (21) over the energy interval $\Delta \omega$, as explained below Eq. (12)], we find the coherent contribution to the partial cross section,

$$
P_{\mathrm{coh}}^{\gamma e}=\left|\sum_{d} \frac{\langle k, \varepsilon|\hat{V}| d\rangle\langle d|\hat{D}| 0\rangle}{E_{0}+\omega-E_{d}+\frac{i}{2} \Gamma_{\mathrm{spr}}}\right|^{2},
$$

the independent-resonance contribution,

$$
\begin{aligned}
& P_{\mathrm{IR}}^{\gamma e}=\frac{D}{2 \pi} \Gamma_{n} \\
& \sum_{b} \frac{|\langle k, \varepsilon|\hat{V}| b\rangle|^{2} \Gamma_{\mathrm{spr}}}{\left(E_{0}+\omega-E_{b}\right)^{2}+\Gamma_{\mathrm{spr}}^{2} / 4} \\
& \times \sum_{d} \frac{|\langle d|\hat{D}| 0\rangle|^{2} \Gamma_{\mathrm{spr}}}{\left(E_{0}+\omega-E_{d}\right)^{2}+\Gamma_{\mathrm{spr}}^{2} / 4},
\end{aligned}
$$

and the residual stochastic contribution,

$$
P_{\text {res }}^{\gamma e}=\frac{D}{2 \pi \Gamma_{n}}\left|\sum_{d} \frac{\langle d|\hat{V}| k, \varepsilon\rangle\langle d|\hat{D}| 0\rangle \Gamma_{\mathrm{spr}}}{\left(E_{0}+\omega-E_{d}\right)^{2}+\Gamma_{\mathrm{spr}}^{2} / 4}\right|^{2} .
$$

In these expressions the matrix elements and sums involve only doorway states. Compound resonances have been eliminated from the sums, and only affect the result through the parameters such as $\Gamma_{\mathrm{spr}}$, the mean level spacing $D$ and the compound state width $\Gamma_{n}$. Similar expressions can be obtained for the probabilities of the photon and electron scattering and electron recombination. These formulae are suitable for the numerical calculations of the resonance-averaged cross sections. Conversion of these equations to the cross sections involves kinematic factors, whose precise form depends on the normalization of the electron continuum states $\varepsilon$ and electromagnetic transition operator $\hat{D}$.

\section{COMPARISON OF THE COHERENT AND STOCHASTIC CONTRIBUTIONS}

\section{A. Photoionization}

Let us compare the magnitudes of the three contributions to the resonance-averaged probability of photoionization $\left(A+\gamma \rightarrow A^{+}+e\right)$, Eqs. (24)-(26). There are two reasons for the possible suppression of the coherent and residual contributions in comparison with the independent-resonance term.
The first point to note is that the basis (doorway) states which contribute to the sums over $b$ and $d$ in the independent-resonance contribution $P_{\mathrm{IR}}^{\gamma e}$, Eq. (25), are in general quite different. The operator $\hat{D}$, which couples the ground state $|0\rangle$ with state $|d\rangle$ is a one-body operator. Hence, the photoabsorption doorway states $d$ are single-electron excitations from the ground state. On the other hand, the two-body Coulomb interaction which couples the final state $|k, \varepsilon\rangle$ with $|b\rangle$, favours dielectronic (doubly-excited) doorway states $b$. The level density of such states is much higher than that of the single-electron excitations. This means that the number of terms which contribute effectively to the sum over $b, N_{b} \sim \Gamma_{\mathrm{spr}} / D_{b}$, is much greater than the number of terms which contribute to the sum over $d, N_{d} \sim \Gamma_{\mathrm{spr}} / D_{d}$ (where $D_{b}$ and $D_{d}$ are the mean spacing between the corresponding doorway states, $D_{b} \ll D_{d}$ ). We thus see that the sum in Eq. (25) contains $\sim N_{b} N_{d}$ positive terms.

In contrast, in both the coherent and residual stochastic parts $P_{\text {coh }}^{\gamma e}$ and $P_{\text {res }}^{\gamma e}$, Eqs. (24) and (26), the same doorway $d$ appears in both matrix elements. As a result, these sums contain $\sim N_{d}^{2}$ terms. Besides this, only $\sim N_{d}$ of these terms (i.e., the diagonal ones) are definitely positive, while the remaining interference terms can have different signs. The expressions for the independent-resonance and the residual stochastic contributions, Eqs. (25) and (26), contain the same prefactors, and we see that the residual contribution is suppressed as $P_{\text {res }}^{\gamma e} / P_{\mathrm{IR}}^{\gamma e} \sim 1 / N_{b}$.

The situation with the coherent contribution is not so simple. According to the above estimates, we have

$$
\frac{P_{\mathrm{ch}}^{\gamma e}}{P_{\mathrm{IR}}^{\gamma e}} \sim \frac{1}{N_{b}} \frac{\Gamma_{n}}{D} \sim \frac{D_{b} \Gamma_{n}}{\Gamma_{\mathrm{spr}} D}
$$

If the compound resonances have a small number of decay channels, then $\Gamma_{n} \ll D$ would normally hold (see Appendix B of Ref. [46] and references therein), and the independent-resonance contribution dominates. However, for the compound states which lie above the ionization threshold, the number of decay channels can be large. In this case one can have $\Gamma_{n} \sim D$ [14], or even observe strongly overlapping resonances with $\Gamma_{n} \gg D$. This means that there could be cases in which the coherent contribution is important.

To illustrate the role of doorways, the photoionization cross section of $\mathrm{Xe}^{q+}$ ions $(q=4-6)$ in the energy range $\omega=90-100 \mathrm{eV}$ is dominated by a prominent narrow maximum due to the $4 d \rightarrow 4 f$ transition [26]. $\mathrm{Xe}^{6+}$ is a closed-shell system, and the $4 d-4 f$ peak in this system appears as a structureless single-particle peak. In the open-shell $\mathrm{Xe}^{5+}$ and $\mathrm{Xe}^{4+}$, the $4 d-4 f$ peak becomes progressively more fragmented, due to mixing between the $4 d-4 f$ doorway and other electronic excitations. 


\section{B. Photon scattering}

For photon scattering $\left(A+\gamma \rightarrow A^{*}+\gamma^{\prime}\right)$, the coherent, independent-resonance, and residual stochastic contributions are obtained by averaging $\left|M_{m}^{\gamma \gamma^{\prime}}\right|^{2}$, where $M_{m}^{\gamma \gamma^{\prime}}$ is given by Eq. (5). The result is given by expressions similar to those in Eqs. (24)-(26):

$$
\begin{aligned}
P_{\mathrm{coh}}^{\gamma \gamma^{\prime}} & =\left|\sum_{d} \frac{\langle m|\hat{D}| d\rangle\langle d|\hat{D}| 0\rangle}{E_{0}+\omega-E_{d}+\frac{i}{2} \Gamma_{\mathrm{spr}}}\right|^{2}, \\
P_{\mathrm{IR}}^{\gamma \gamma^{\prime}} & =\frac{D}{2 \pi \Gamma_{n}} \sum_{b} \frac{|\langle m|\hat{D}| b\rangle|^{2} \Gamma_{\mathrm{spr}}}{\left(E_{0}+\omega-E_{b}\right)^{2}+\Gamma_{\mathrm{spr}}^{2} / 4} \\
& \times \sum_{d} \frac{|\langle d|\hat{D}| 0\rangle|^{2} \Gamma_{\mathrm{spr}}}{\left(E_{0}+\omega-E_{d}\right)^{2}+\Gamma_{\mathrm{spr}}^{2} / 4}, \\
P_{\mathrm{res}}^{\gamma \gamma^{\prime}} & =\frac{D}{2 \pi \Gamma_{n}}\left|\sum_{d} \frac{\langle d|\hat{D}| m\rangle\langle d|\hat{D}| 0\rangle \Gamma_{\mathrm{spr}}}{\left(E_{0}+\omega-E_{d}\right)^{2}+\Gamma_{\mathrm{spr}}^{2} / 4}\right|^{2} .
\end{aligned}
$$

For elastic $(m=0)$ or weakly inelastic scattering (e.g., when the final state $m$ belongs to the same electronic configuration as the initial state 0 ), same doorways $d$ will be available in the sums for $P_{\mathrm{coh}}^{\gamma \gamma^{\prime}}$ and $P_{\mathrm{res}}^{\gamma \gamma^{\prime}}$, so that the latter is suppressed as $1 / N_{d}$ relative to $P_{\mathrm{IR}}^{\gamma \gamma^{\prime}}$. Simple single-electron excitation doorways do not have a dense spectrum, which means that $N_{d}$ may be small, making all three contributions comparable.

On the other hand, if the energy of the incident photon is sufficiently large, inelastic (Raman) photon scattering becomes much more prominent due to the availability of many excited final states $m$. The majority of them will share no or few doorways with the initial state 0 , which means that both $P_{\text {coh }}^{\gamma \gamma^{\prime}}$ and $P_{\text {res }}^{\gamma \gamma^{\prime}}$ will be strongly suppressed in comparison with $P_{\mathrm{IR}}^{\gamma \gamma^{\prime}}$. The same conclusion is true if we consider the total photon scattering cross section summed over the final states $m$.

To make the comparison clearer, we can present our results in a conventional Breit-Wigner form by replacing the squared matrix elements by the corresponding partial widths. For example, the coherent contribution (28) to the total photon scattering cross section is

$$
\begin{aligned}
P_{\mathrm{coh}}^{\gamma \gamma^{\prime}} & =\sum_{d m} \frac{|\langle m|\hat{D}| d\rangle|^{2}|\langle d|\hat{D}| 0\rangle|^{2}}{\left(E_{0}+\omega-E_{d}\right)^{2}+\Gamma_{\mathrm{spr}}^{2} / 4} \\
& \propto \sum_{d} \frac{\Gamma_{d \rightarrow 0}^{(r)} \Gamma_{d}^{(r)}}{\left(E_{0}+\omega-E_{d}\right)^{2}+\Gamma_{\mathrm{spr}}^{2} / 4}
\end{aligned}
$$

where the total radiative width of the doorway state $d$ is

$$
\Gamma_{d}^{(r)} \propto \sum_{m}|\langle d|\hat{D}| m\rangle|^{2} \approx \sum_{e}|\langle d|\hat{D}| e\rangle|^{2} .
$$

In Eq. (31) we neglected the interference terms between different doorway states in (28), since their contribution is strongly suppressed $\left(\sim N_{m}^{-1 / 2}\right)$ after summation over the large number $N_{m}$ of compound states $m$ populated after the emission of the final-state photon. Note also that we have replaced the sum over the compound states in the total radiative width (33) by the sum over the basis states $e$, owing to normalization $\sum_{m}\left|C_{e}^{(m)}\right|^{2}=1$. As a result, the final expression in Eq. (32) includes only the matrix elements between doorway states and relatively simple states $|0\rangle$ and $|e\rangle$.

Equation (32) describes the Breit-Wigner-like contributions of the doorway states $d$ to the coherent part of the photon scattering cross section. Comparing with the total cross section (16), we see that the coherent contribution is suppressed by the ratio $\Gamma_{d}^{(r)} / \Gamma_{d} \approx \Gamma_{d}^{(r)} / \Gamma_{\text {spr }} \ll 1$. In a similar way, the independent-resonance contribution can be written as

$$
P_{\mathrm{IR}}^{\gamma \gamma^{\prime}} \propto \frac{\Gamma_{n}^{(r)}}{\Gamma_{n}} \sum_{d} \frac{\Gamma_{d \rightarrow 0}^{(r)} \Gamma_{\mathrm{spr}}}{\left(E_{0}+\omega-E_{d}\right)^{2}+\Gamma_{\mathrm{spr}}^{2} / 4},
$$

where

$$
\Gamma_{n}^{(r)}=\sum_{m} \Gamma_{n \rightarrow m}^{(r)}=\sum_{m b} \overline{C_{b}^{(n)^{2}}} \Gamma_{b \rightarrow m}^{(r)}
$$

[cf. Eqs. (25) and (22)]. Compared to the total cross section (in which $\Gamma_{d} \approx \Gamma_{\mathrm{spr}}$ ), the independent-resonance contribution Eq. (34) contains an extra factor $\Gamma_{n}^{(r)} / \Gamma_{n}$, which is the branching ratio for the radiative decay of the resonances. Since $\Gamma_{n}^{(r)} \sim \Gamma_{d}^{(r)}$ (for the doorways represented in $n$ ), the ratio $P_{\mathrm{coh}}^{\gamma \gamma^{\prime}} / P_{\mathrm{IR}}^{\gamma \gamma^{\prime}} \sim \Gamma_{n} / \Gamma_{\mathrm{spr}} \ll 1$, i.e., the coherent contribution is suppressed in comparison with the the independent-resonance term.

\section{Electron scattering}

Considering electron scattering $\left(A+e \rightarrow A^{*}+e^{\prime}\right)$, the three contributions to the resonance-averaged cross section are

$$
\begin{aligned}
P_{\mathrm{coh}}^{e e^{\prime}} & =\left|\sum_{d} \frac{\left\langle k, \varepsilon^{\prime}|\hat{V}| d\right\rangle\langle d|\hat{V}| i, \varepsilon\rangle}{E_{i}+\varepsilon-E_{d}+\frac{i}{2} \Gamma_{\mathrm{spr}}}\right|^{2}, \\
P_{\mathrm{IR}}^{e e^{\prime}} & =\frac{D}{2 \pi \Gamma_{n}} \sum_{b} \frac{\left|\left\langle k, \varepsilon^{\prime}|\hat{V}| b\right\rangle\right|^{2} \Gamma_{\mathrm{spr}}}{\left(E_{i}+\varepsilon-E_{b}\right)^{2}+\Gamma_{\mathrm{spr}}^{2} / 4} \\
& \times \sum_{d} \frac{|\langle d|\hat{V}| i, \varepsilon\rangle|^{2} \Gamma_{\mathrm{spr}}}{\left(E_{i}+\varepsilon-E_{d}\right)^{2}+\Gamma_{\mathrm{spr}}^{2} / 4}, \\
P_{\mathrm{res}}^{e e^{\prime}} & =\frac{D}{2 \pi \Gamma_{n}}\left|\sum_{d} \frac{\left\langle d|\hat{V}| k, \varepsilon^{\prime}\right\rangle\langle d|\hat{V}| i, \varepsilon\rangle \Gamma_{\mathrm{spr}}}{\left(E_{i}+\varepsilon-E_{d}\right)^{2}+\Gamma_{\mathrm{spr}}^{2} / 4}\right|^{2} .
\end{aligned}
$$

For low incident electron energies, one can only have elastic or quasielastic scattering, when state $k$ is identical or similar to $i$. In this case the suppression of the coherent and residual contributions is $\sim 1 / N_{d}$. However, the doorways involved in electron capture and re-emission 
are dielectronic excitations of the compound atom or ion. Their level density is higher than that of single-electron excitations, leading to greater values of $N_{d}$ and stronger suppression than in photon scattering. At higher incident electron energies more final states $k$ become available. Such states will have fewer common doorways with the initial states and the relative importance of the $P_{\mathrm{IR}}^{e e^{\prime}}$ will increase further.

\section{Electron-ion recombination}

Last but not least, electron recombination $\left(A^{q+}+e \rightarrow\right.$ $\left.A^{(q-1)+}+\gamma\right)$ is quite special. For complex targets, many final states (channels) are available even at the lowest incident electron energy [50]. To obtain the total recombination cross section, one needs to sum over all final states $m$ of the ion $A^{(q-1)+}$. The three contributions to the reaction probability then are

$$
\begin{aligned}
P_{\mathrm{coh}}^{e \gamma} & =\sum_{m}\left|\sum_{d} \frac{\langle m|\hat{D}| d\rangle\langle d|\hat{V}| i, \varepsilon\rangle}{E_{i}+\varepsilon-E_{d}+\frac{i}{2} \Gamma_{\mathrm{spr}}}\right|^{2}, \\
P_{\mathrm{IR}}^{e \gamma} & =\frac{D}{2 \pi \Gamma_{n}} \sum_{m} \sum_{b} \frac{|\langle m|\hat{D}| b\rangle|^{2} \Gamma_{\mathrm{spr}}}{\left(E_{i}+\varepsilon-E_{b}\right)^{2}+\Gamma_{\mathrm{spr}}^{2} / 4} \\
& \times \sum_{d} \frac{|\langle d|\hat{V}| i, \varepsilon\rangle|^{2} \Gamma_{\mathrm{spr}}}{\left(E_{i}+\varepsilon-E_{d}\right)^{2}+\Gamma_{\mathrm{spr}}^{2} / 4}, \\
P_{\mathrm{res}}^{e \gamma} & =\frac{D}{2 \pi \Gamma_{n}} \sum_{m}\left|\sum_{d} \frac{\langle d|\hat{D}| m\rangle\langle d|\hat{V}| i, \varepsilon\rangle \Gamma_{\mathrm{spr}}}{\left(E_{i}+\varepsilon-E_{d}\right)^{2}+\Gamma_{\mathrm{spr}}^{2} / 4}\right|^{2} .
\end{aligned}
$$

For the majority of states $m$, the doorways $b$ and $d$ in the two matrix elements in Eq. (40) will be different. This means that both the coherent and the residual contributions, in which $b=d$, are strongly suppressed relative to the independent-resonance contribution.

Similarly to Eq. (34), the recombination probability $P_{\mathrm{IR}}^{e \gamma}$ can be written in terms of the widths,

$$
P_{\mathrm{IR}}^{e \gamma} \propto \frac{\Gamma_{n}^{(r)}}{\Gamma_{n}} \sum_{d} \frac{\Gamma_{d \rightarrow i}^{(a)} \Gamma_{\mathrm{spr}}}{\left(E_{i}+\varepsilon-E_{d}\right)^{2}+\Gamma_{\mathrm{spr}}^{2} / 4} .
$$

This large ratio of the number of open channels for photon- and electron-emission reactions at low incident electron energies also explains the enhancement of the fluorescent yield in the resonant electron capture up to nearly $100 \%$ (i.e., $\Gamma_{n}^{(r)} / \Gamma_{n} \approx 1$ ) [5, 14-16]. In this case electron recombination dominates in the total cross section Eq. (17) of the electron collisions with highly charged ions, and the total cross section can be calculated using the IR stochastic contribution only. On the other hand, the total cross section is expressed via the imaginary part of the coherent elastic amplitude [cf. Eq. (15)]. Thus, the optical theorem establishes a relation between the coherent and stochastic contributions.

To illustrate the effect of the fluorescence yield, Fig. 2 shows the calculated total resonant capture rate and the photorecombination rate for electron collisions with $W^{20+}$. Working equations for the process of electron-ion recombination, in which the quantities of interest are expressed in terms of two-particle radial Coulomb integrals, angular momentum algebra coefficients and sums over single-particle states, can be found in Refs. [14-16]. The small difference between the resonant capture rate from Ref. [15] (calculated for $\varepsilon=1 \mathrm{eV}$ and plotted assuming $1 / \varepsilon$ energy dependence of the cross section) and [16] is due to a slightly different numerical procedure. Suppression of the recombination rate with respect to the total resonant capture rate, due to the factor $\Gamma_{n}^{(r)} / \Gamma_{n}$, is clearly visible (see Refs. $[15,16]$ for the working equations used). The difference in the detailed energy dependence of the calculated and measured recombination rate is likely due to inaccurate energies of the dielectronic doorways in the calculation.

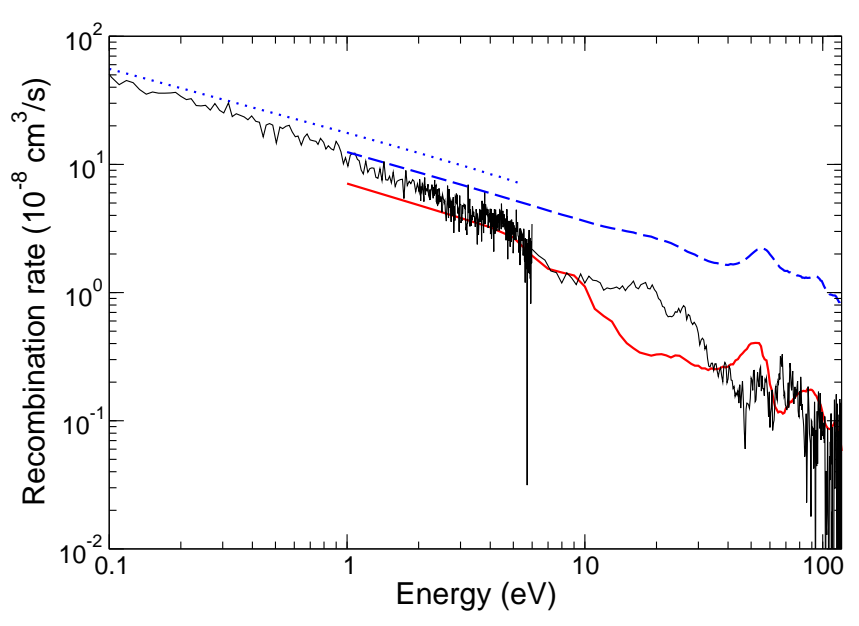

FIG. 2. (Color online) The graph shows the total rate of electron resonant capture by $\mathrm{W}^{20+}$, calculated using statistical theory (dotted blue line, Ref. [15]; dashed blue line, Ref. [16]), and the calculated recombination rate (thick solid red line), obtained by including the effect of the nonunit fluorescence yield [16]. Thin solid black line is the experimental recombination rate from Ref. [20].

It is interesting that the same mechanism that leads to strongly enhanced recombination in $A^{q+}+e$ collisions, should strongly suppress photoionization of $A^{(q-1)+}$ at photon energies close to threshold. Re-writing the corresponding IR contribution Eq. (25) in terms of widths,

$$
P_{\mathrm{IR}}^{\gamma e} \propto \frac{\Gamma_{n}^{(a)}}{\Gamma_{n}} \sum_{d} \frac{\Gamma_{d \rightarrow 0}^{(r)} \Gamma_{\mathrm{spr}}}{\left(E_{0}+\omega-E_{d}\right)^{2}+\Gamma_{\mathrm{spr}}^{2} / 4},
$$

where $\Gamma_{n}^{(a)}$ is the total autoionization width of state $n$, we see that in ions such as $\mathrm{Au}^{24+}$ or $\mathrm{W}^{19+}$, in which $\Gamma_{n}^{(r)}>\Gamma_{n}^{(a)}$ near threshold, Raman scattering, Eq. (34), will be favoured over ionization. A possible way of observing this effect in experiment is to measure and compare the total photoabsorption and photoionization cross sections. Alternatively, one can measure the spectrum of 
secondary photon from the resonant Raman scattering, varying the primary photon energy across the ionization threshold.

\section{CONCLUSIONS}

In this work we have investigated the role of doorway states in electron- and photon-induced reactions mediated by strongly mixed compound resonances. Our analysis shows that the resonance-averaged total reaction cross sections $\sigma_{\text {tot }}$ are given by the coherent contributions of the compound resonances. These cross sections are expressed in terms of doorway resonances (i.e., simple states coupled directly to the initial state of the target). The only difference with the standard approach for dielectronic recombination and photoionization is that the doorway resonances are broadened by the spreading width $\Gamma_{\text {spr }}$, which described their coupling to the dense spectrum of chaotic compound states.

The situation with the partial cross sections is more complicated. For processes such as radiative electron capture (photorecombination) or photon scattering at energies which place the system in the strong-mixing regime, the number of the decay channels is very large. As a result, the stochastic contribution, corresponding to the independent-resonance approximation dominates. The (resonance-averaged) partial cross section can then be calculated from the total cross section by including the appropriate branching ratio, e.g., $\sigma_{r}^{e}=\left(\overline{\Gamma_{n}^{(r)}} / \overline{\Gamma_{n}}\right) \sigma_{\text {tot }}^{e}$, for photorecombination. Here the ratio of the radiative and total widths of the compound resonances and the electron capture cross section $\sigma_{\text {tot }}^{e}$ can be calculated as in Ref. [16]. On the other hand, if the process leads to electron emission (photoionization, electron scattering) and the number of decay channels is small (i.e., the energy does not exceed threshold by much), the independentresonance approximation may be deficient and the coherent contribution may need to be included.

\section{ACKNOWLEDGMENTS}

This work was supported by the Australian Research Council and Russian Foundation for Basic Research Grant No. 14-02-00241. We thank J. Berengut, V. Dzuba and C. Harabati for useful discussions.
[1] T. Guhr, A. Müller-Groeling, and H. A. Weidenmüller, Phys. Rep. 299, 189 (1998).

[2] V. Zelevinsky, B. A. Brown, N. Frazier, and M. Horoi, Phys. Rep. 276, 85 (1996).

[3] H. A. Weidenmüller and G. E. Mitchell, Rev. Mod. Phys. 81, 539 (2009).

[4] V. V. Flambaum, A. A. Gribakina, G. F. Gribakin, and M. G. Kozlov, Phys. Rev. A 50, 267 (1994).

[5] G. F. Gribakin, A. A. Gribakina, and V. V. Flambaum, Aust. J. Phys. 52, 443 (1999), physics/9811010.

[6] G. F. Gribakin and S. Sahoo, J. Phys. B 36, 3349 (2003).

[7] K. K. Lehmann, G. Scoles, and B. H. Pate, Annu. Rev. Phys. Chem. 45, 241 (1994).

[8] D. J. Nesbitt and R. W. Field, J. Phys. Chem. 100, 12735 (1996).

[9] M. Gruebele and R. Bigwood, Int. Rev. Phys. Chem. 17, 91 (1998).

[10] A. A. Makarov, A. L. Malinovsky, and E. A. Ryabov, Physics-Uspekhi 55, 977 (2012).

[11] A. Frisch, M. Mark, K. Aikawa, F. Ferlaino, J. L. Bohn, C. Makrides, A. Petrov, and S. Kotochigova, Nature 507, 475 (2014).

[12] O. P. Sushkov and V. V. Flambaum, Sov. Phys. Uspekhi 25, 1 (1982), [Usp. Fiz. Nauk 136, 3 (1982)].

[13] V. V. Flambaum and G. F. Gribakin, Prog. Part. Nucl. Phys. 35, 423 (1995).

[14] V. V. Flambaum, A. A. Gribakina, G. F. Gribakin, and C. Harabati, Phys. Rev. A 66, 012713 (2002).

[15] V. A. Dzuba, V. V. Flambaum, G. F. Gribakin, and C. Harabati, Phys. Rev. A 86, 022714 (2012).
[16] V. A. Dzuba, V. V. Flambaum, G. F. Gribakin, C. Harabati, and M. G. Kozlov, Phys. Rev. A 88, 062713 (2013).

[17] O. Uwira, A. Müller, W. Spies, J. Linkemann, A. Frank, T. Cramer, L. Empacher, R. Becker, M. Kleinod, P. Mokler, J. Kenntner, A. Wolf, U. Schramm, T. Schüssler, D. Schwalm, and D. Habs, Hyperfine Interact. 99, 295 (1996).

[18] D. M. Mitnik, M. S. Pindzola, F. Robicheaux, N. R. Badnell, O. Uwira, A. Müller, A. Frank, J. Linkemann, W. Spies, N. Angert, P. H. Mokler, R. Becker, M. Kleinod, S. Ricz, and L. Empacher, Phys. Rev. A 57, 4365 (1998).

[19] A. Hoffknecht, O. Uwira, S. Schennach, A. Frank, J. Haselbauer, W. Spies, N. Angert, P. H. Mokler, R. Becker, M. Kleinod, S. Schippers, and A. Müller, J. Phys. B 31, 2415 (1998).

[20] S. Schippers, D. Bernhardt, A. Müller, C. Krantz, M. Grieser, R. Repnow, A. Wolf, M. Lestinsky, M. Hahn, O. Novotný, and D. W. Savin, Phys. Rev. A 83, 012711 (2011).

[21] N. R. Badnell, C. P. Ballance, D. C. Griffin, and M. O'Mullane, Phys. Rev. A 85, 052716 (2012).

[22] K. Spruck, N. R. Badnell, C. Krantz, O. Novotný, A. Becker, D. Bernhardt, M. Grieser, M. Hahn, R. Repnow, D. W. Savin, A. Wolf, A. Müller, and S. Schippers, Phys. Rev. A 90, 032715 (2014).

[23] Martins, M., Sladeczek, P., Tiedtke, K., and Zimmermann, P., Eur. Phys. J. D 1, 47 (1998).

[24] G. O'Sullivan, P. K. Carroll, P. Dunne, R. Faulkner, C. McGuinness, and N. Murphy, J. Phys. B 32, 1893 (1999). 
[25] M. Lysaght, D. Kilbane, N. Murphy, A. Cummings, P. Dunne, and G. O'Sullivan, Phys. Rev. A 72, 014502 (2005).

[26] A. Aguilar, J. D. Gillaspy, G. F. Gribakin, R. A. Phaneuf, M. F. Gharaibeh, M. G. Kozlov, J. D. Bozek, and A. L. D. Kilcoyne, Phys. Rev. A 73, 032717 (2006).

[27] M. G. Su, C. Z. Dong, N. Murphy, and G. O'Sullivan, Phys. Rev. A 79, 042507 (2009).

[28] M. Habibi, D. A. Esteves, R. A. Phaneuf, A. L. D. Kilcoyne, A. Aguilar, and C. Cisneros, Phys. Rev. A 80, 033407 (2009).

[29] S. Schippers, S. Ricz, T. Buhr, A. Borovik, J. Hellhund, K. Holste, K. Huber, H.-J. Schäfer, D. Schury, S. Klumpp, K. Mertens, M. Martins, R. Flesch, G. Ulrich, E. Rühl, T. Jahnke, J. Lower, D. Metz, L. P. H. Schmidt, M. Schöffler, J. B. Williams, L. Glaser, F. Scholz, J. Seltmann, J. Viefhaus, A. Dorn, A. Wolf, J. Ullrich, and A. Müller, J. Phys. B 47, 115602 (2014).

[30] L. G. Gerchikov and G. F. Gribakin, Phys. Rev. A 77, 042724 (2008).

[31] G. F. Gribakin, J. A. Young, and C. M. Surko, Rev. Mod. Phys. 82, 2557 (2010).

[32] V. V. Flambaum, Physica Scripta T 46, 198 (1993).

[33] V. V. Flambaum and O. K. Vorov, Phys. Rev. Lett. 70, 4051 (1993).

[34] V. V. Flambaum, A. A. Gribakina, and G. F. Gribakin, Phys. Rev. A 58, 230 (1998).

[35] V. V. Flambaum, A. A. Gribakina, G. F. Gribakin, and I. V. Ponomarev, Phys. Rev. E 57, 4933 (1998).

[36] V. V. Flambaum, A. A. Gribakina, G. F. Gribakin, and I. V. Ponomarev, Physica D 131, 205 (1999).

[37] V. V. Flambaum and G. F. Gribakin, Philos. Mag. B 80, 2143 (2000).

[38] Y. Yamada, T. Ebata, M. Kayano, and N. Mikami, J. Chem. Phys. 120, 7400 (2004).

[39] P. Glans, E. Lindroth, N. Eklöw, W. Zong, G. Gwinner, A. Saghiri, M. Pajek, H. Danared, and R. Schuch,
Nucl. Instrum. Methods B 154, 97 (1999).

[40] E. Lindroth, H. Danared, P. Glans, Z. Pešić, M. Tokman, G. Vikor, and R. Schuch, Phys. Rev. Lett. 86, 5027 (2001).

[41] It is interesting to compare the resolution achieved in the state-of-the-art electron-ion recombination measurements with that possible in the studies of molecular spectra. In the former case, the resolution is limited by the temperature of the electron beam, which can be as low as 1-10 meV [39, 40, 51], which gives the relative precision of $10^{-5}$, when compared with the excitation energy of the ion (e.g., $\mathrm{Au}^{24+}$ or $\mathrm{W}^{19+}$ ) formed in the recombination event. In contrast, the frequency spread of $10^{-4} \mathrm{~cm}^{-1}$ achieved in high-resolution molecular spectroscopy [43], is about $10^{-8}$ of the vibrational energy probed, so the experiment is capable of detecting individual "bath" states.

[42] A. A. Stuchebrukhov and R. A. Marcus, J. Chem. Phys. 98, 6044 (1993).

[43] A. McIlroy and D. J. Nesbitt, J. Chem. Phys. 92, 2229 (1990).

[44] H. S. W. Massey and D. R. Bates, Reports on Progress in Physics 9, 62 (1942).

[45] H. Feshbach, Ann. Phys. 19, 287 (1962).

[46] V. V. Flambaum, A. A. Gribakina, and G. F. Gribakin, Phys. Rev. A 54, 2066 (1996).

[47] B. L. Altshuler, Y. Gefen, A. Kamenev, and L. S. Levitov, Phys. Rev. Lett. 78, 2803 (1997).

[48] L. D. Landau and E. M. Lifshitz, Quantum Mechanics, 3rd ed., Vol. 3 (Pergamon, Oxford, 1977).

[49] M. S. Pindzola, N. R. Badnell, and D. C. Griffin, Phys. Rev. A 46, 5725 (1992).

[50] The number of finite states is infinite even for simple target ions, as they include Rydberg states for the incident electron. However, this is a feature of the single-electron dynamics, and it does not lead to enhancements related to many-electron excitations produced in complex systems.

[51] A. Müller, Int. J. Mass Spectr. 192, 9 (1999). 